\title{
Quantitative evaluation method of arc sound spectrum based on sample entropy
}

\author{
Ping Yao ${ }^{\mathrm{a}, \mathrm{b}}$, Kang Zhou ${ }^{\mathrm{c}, *}$, Qiang Zhu ${ }^{\mathrm{d}}$ \\ a School of Electrical and Mechanical, Guangdong Polytechnic Normal University, China \\ ${ }^{\mathrm{b}}$ School of Mechanical and Automotive Engineering, South China University of Technology, Guangzhou, China \\ ' State Key Laboratory of High-temperature Gas Dynamics, Institute of Mechanics, Chinese Academy of Sciences, China \\ ${ }^{\mathrm{d}}$ School of Electronic and Communication Engineering, Guangdong Communication Polytechnic, China
}

\section{A R T I C L E I N F O}

\section{Article history:}

Received 19 April 2016

Received in revised form 4 October 2016

Accepted 11 January 2017

Available online 9 February 2017

\section{Keywords:}

Arc sound signal

Sample entropy

PSD

ASSE

\begin{abstract}
A B S T R A C T
Arc sound analysis is an effective way to evaluate the stability of the arc welding process. Current methods cannot effectively quantify the disorder of the process. By studying the characteristics of the arc sound signal, we found that low frequency random mutation of arc sound power resulted from unstable factors, such as splashes or short circuits, increased the complexity and randomness of the arc sound signals. Then the arc sound signals were visualized on time-frequency interface by means of spectrogram, and it was found that the max power spectral density (PSD) distribution of spectrogram was closely related to the stability of arc welding process. Moreover, a method based on sample entropy was proposed to further quantify the relation. Finally, considering the factors such as averages of max PSD and the standard deviations of sample entropy, a compound quantitative evaluation indicator, arc sound sample entropy (ASSE), which can avoid the influence of different parameters on the quantitative results, was proposed, so that the stability of arc welding process can be quantitatively presented. Testing results showed that the accuracy rate of the method was more than 90 percent.
\end{abstract}

(c) 2017 Elsevier Ltd. All rights reserved.

\section{Introduction}

The stability of arc welding process is an important condition for assuring the quality of welding products. For decades, a lot of relative works were conducted to monitor the stability of welding process. Luksa et al. [1] online collected the process signals by an experimental platform, and then analyzed the welding quality by signal variations. Hermans et al. [2] also collected electrical signals during the welding process and analyzed the short circuit frequency, and then concluded that the welding process was stable when the short circuit frequency was the same as the frequency of weld pool oscillations. Adolfsson et al. [3] employed a repeated sequential probability ratio test (SPRT) algorithm to detect the sudden minor changes of weld voltage, and then concluded that it was possible to online detect changes in weld quality automatically. Though there were relative works considering the stability of welding process, those methods could only detect some abnormal phenomena during the welding process, instead of analyzing, especially quantifying the stability of a whole welding process. In addition, arc welding process is a highly nonlinear and multi-field coupled process, limited electrical signals cannot sufficiently

\footnotetext{
* Corresponding author.

E-mail address: Zhoukang326@126.com (K. Zhou).
} 
present the characteristics. Hence, other signals which include abundant information of the process should be introduced to evaluate the stability, and then to help to achieve high quality welding production.

Arc sound signals contain a large amount of information generated during arc welding process [4-6]. Skilled welders were able to determine the stability of the welding process by means of arc sound alone. Cudina and Prezelj [7] studied the feasibility of online monitoring welding quality by arc sound in Gas Metal Arc Welding(GMAW), and thought that irregular changes of arc sounds were the reaction of the unstability of welding process. Liu et al. [6] extracted the arc sound features from four typical weld penetration statuses and analyzed the relation between weld penetration statuses and arc sound characteristics. Pal et al. [8] acquired current, voltage and arc sound signals synchronously and analyzed the correlation between electrical signals and arc sound signals in terms of time-domain and frequency-domain. Grad et al. [9] thought that the irregular of arc sound signal reflected the unstability of welding process. Hence, the arc sound signal is closely relative to the stability of arc welding process, and it can be employed to evaluate the stability of the process.

For decades, arc sound signals were usually described by time-domain, frequency spectrogram and power spectrogram. In such a case, the relation between time, frequency and power spectral density cannot be simultaneously presented and employed. Saini and Floyd [10] monitored weld quality online by kinds of indicators in time-domain and frequencydomain of the arc sound, and then proposed that the advantages of time-domain and frequency-domain should be combined, since the two analyses had their own advantages and disadvantages, no one could sufficiently show the signal characteristics alone.

Under this circumstance, sound spectrum analysis technology was developed. A spectrogram, or sonogram, is a visual technology, which can be employed to represent the variations of sound or other signals with time or other selected variables. In this work, spectrogram, which is also the sound spectrum figure, is a colorful plane figure obtained from spectral decomposition for sound signals. In the figure, time is in the x-axis, while frequency is in the y-axis, and each value of pixel denotes the power spectral density (PSD) of the signal at designated time and frequency. The information of signal stability could be obtained via sound spectrum analysis technology, due to varying information of the power spectral density and frequency waveform can be observed from the figure.

The spectrogram is one of the best-known time-frequency distributions suitable for analyzing signals whose power varies both in time and frequency. It was commonly employed to phonetically identify spoken words [11,12], or analyze various callings of animals [13]. Also, this tool was used extensively in the development of polyphonic piano transcription [14], sonar, radar, and speech processing $[15,16]$, and so on.

According to corresponding researches and applications, it is feasible to employ the sound spectrum analysis technology to analyze the arc sound signal, and then obtain the information about stability during the process. However, due to the information in sound spectrum figure is in pixel form, and the information acquisition is determined by analyses' experiences and interpretation standards, large errors may be obtained during the process. In addition, the figure information obtained may have low repeatability, so that it is hard to analyze and after-treatment. These disadvantages seriously restrict the application of arc sound spectrum analyzing technology.

Entropy is a mathematical variable which can describe the stability of a system. It is extensively used to calculate phenomena of system disorder, that is, the degree of confusion of a system. Approximate entropy is proposed by Pincus et al [17] to overcome the difficulty in solving the entropy in chaos. Approximate entropy is mainly employed to measure the probability of new model generated in the signal. The more complex the sequence, the greater the probability of generating a new model and the corresponding approximate entropy [18,19]. Approximate entropy was initially developed to analyze medical data, such as heart rate [18], and later its applications were spread to other areas, such as finance [20], psychology [21], and human factors engineering [22].

Recently, researchers have made some welding process stability studies by means of approximate entropy. Tolle et al. [23] found that the approximate entropy of arc voltage increased with the increasing of wire feed speed in GMAW. Cao et al. [24,25] proposed that approximate entropy can be used to quantify arc and welding process stability in shortcircuiting GMAW. Nie et al. [26] used neural network to predict the approximate entropy of pulsed metal inert gas(MIG) welding of aluminum alloy and achieved good results. Zhang et al. [27] evaluated the effect of adaptive control by approximate entropy and thought that the smaller the approximate entropy, the better the adaptive control. The work showed that the approximate entropy and stability of welding signals have a certain relation but relatively poor consistency due to effects of data length and embedding dimension. Richman et al. [28] proposed a new time-series complexity measuring method on the basis of comparison between approximate entropy and sample entropy, and then concluded that the sample entropy can achieve better consistency and accuracy with faster computing speed than that of approximate entropy, because sample entropy excludes self-match values, and reduces approximate entropy errors.

So far, arc sound detections and corresponding researches were in analyzing stage instead of actual application, and the researches focused on exploring the arc sound characteristics and the relation between arc sound and welding quality. There was little work which employed the arc sound to quantitatively analyze the stability of welding process. Under this circumstance, at first, we collected the original arc sound signal by proposed experimental platform and processed the data using wavelet packet filtering tool, and then analyzed the corresponding sound spectrum figure using the sample entropy algorithm. Finally, the stability information of arc welding process can be quantitatively collected. In this work, we aim to explore a new method and design the quantitative indicators of evaluating arc sound stability of welding process by spectrogram and sample entropy. The work can achieve the goal that the stability of arc welding process can be effectively quantified. 


\section{Arc sound signals pretreatment and characteristics analysis}

In this work, arc sound signals were collected by our self-developed synchronous multi-information acquisition device [29], the sensor employed AWA 14,423 sound conducting apparatus, which was an industrial sound sensor. Its resolution was $50 \mathrm{mV} / \mathrm{Pa}$, the frequency response was between $10 \mathrm{~Hz}$ and $20 \mathrm{kHz}$ and the dynamic range was between $20 \mathrm{~dB}$ and $140 \mathrm{~dB}$. The actual sampling frequency in this work was $44.1 \mathrm{kHz}$. In this device, a self-developed welding inverter was employed for double wire pulsed MIG welding. Q235 steel with thickness of $8.0 \mathrm{~mm}$ was used as bead on plate welds. Welding wire was H08Mn2Si with $1.0 \mathrm{~mm}$ of diameter. The shielding gas was pure argon with $15 \mathrm{~L} / \mathrm{min}$ of flow rate. The integrated experimental platform was shown in Fig. 1.

Arc sound signal was shown in Fig. 2(a). Because various noises were included in the signal, it was difficult to distinguish the pure signals characteristics from the integrated information. To reduce the noises, the collected arc sound signals were pretreated by wavelet packet threshold filtering. According to arc sound signal characteristics, the threshold was adjusted to an appropriate size to make noise as few as possible for further processing.

Pretreated signals by wavelet packet threshold filtering were shown in Fig. 2(b). It could be found that the most noises were eliminated after wavelet packet pretreatment. Signals were significantly clearer than before. It can be found that the voltage amplitude in Fig. 2(b) was lower than that in Fig. 2(a). Between $0.28 \mathrm{~s}$ and $0.9 \mathrm{~s}$, the voltage amplitude of original signal in Fig. 2(a) varied from $-0.4 \mathrm{~V}$ to $0.4 \mathrm{~V}$, while the variation range was from $-0.3 \mathrm{~V}$ to $0.3 \mathrm{~V}$ in Fig. 2(b). In addition, there were significant changes occurred at between $0.2 \mathrm{~s}$ and $0.22 \mathrm{~s}$, as well as at $0.9 \mathrm{~s}$, and these characteristic changes were much clearer in Fig. 2(b) than that in Fig. 2(a). Interferences nearby $0.1 \mathrm{~s}, 0.22 \mathrm{~s}$ and $0.9 \mathrm{~s}$ had been saved. After listened to the noise-reduced arc sounds, it could be found that the sounds were clearer, and "poop" sounds while splashing were still retained, which showed that the signal characteristics were well preserved after noise reduction by wavelet packet threshold filtering.

The power spectrum density(PSD) represented on a logarithmic scale was obtained after Fast Fourier Transform(FFT) for arc sound signals [30,31], as shown in Fig. 2(c). It could be seen that arc sound frequencies were generally less than $4000 \mathrm{~Hz}$, and the most power was concentrated within the frequency range from 0 and $2000 \mathrm{~Hz}$. The power per hertz above $2000 \mathrm{~Hz}$ was quickly lowered.

In order to cope with the situation that the limitation of traditional analysis can only be used in the time-domain and frequency-domain occasions, signals were windowed to determine the overall spectrum variation with time by ShortTime Fourier Transform (STFT). Since stability analysis of the welding process focuses on splash and other factors, the time resolution is very important. Hamming window [32] was chosen in this work, whose length $N$ was 201.

After STFT for the arc sound signals shown in Fig. 2(a), the result was shown in Fig. 3, which was a three-dimensional (3D) figure. The peaks represented signal voltage mutation, meaning that the splashes occurred under the designated frequency and time. The first remarkable splash occurred between $0.05-0.1 \mathrm{~s}$, and the corresponding frequency center was $1400 \mathrm{~Hz}$, the peak amplitude of signal voltage was around $0.25-0.3 \mathrm{~V}$, while the second large splash appeared at $0.22 \mathrm{~s}$, which was consistent with Fig. 2(a); the arc sound was relatively stable between $0.4 \mathrm{~s}$ and $0.8 \mathrm{~s}$, and no peak appeared during this time, moreover, the corresponding voltage was about $0.1 \mathrm{~V}$ or less. In addition, there were few peaks occurred around $0.9 \mathrm{~s}$, the voltage amplitude might about $0.2 \mathrm{~V}$.

The corresponding spectrograms of arc sound signals in Fig. 2(a) were shown in Fig. 4. The x-axis denoted the time, while the $y$-axis denoted the frequency, and each pixel denoted PSD of the signal at designated time and frequency. Hence, any PSD changes lead the color to correspondingly change. As shown from the color bar, the deeper the color, the higher the PSD of the point. As can be seen from the figure, the frequencies of higher PSD were mainly concentrated within $2000 \mathrm{~Hz}$, in less than $0.2 \mathrm{~s}$, there were some messy high PSD distributions, which meant that there might be more uncertain noises during this time. The PSD distribution between $0.4 \mathrm{~s}$ and $0.8 \mathrm{~s}$ was uniform and regular, and then the corresponding colors were

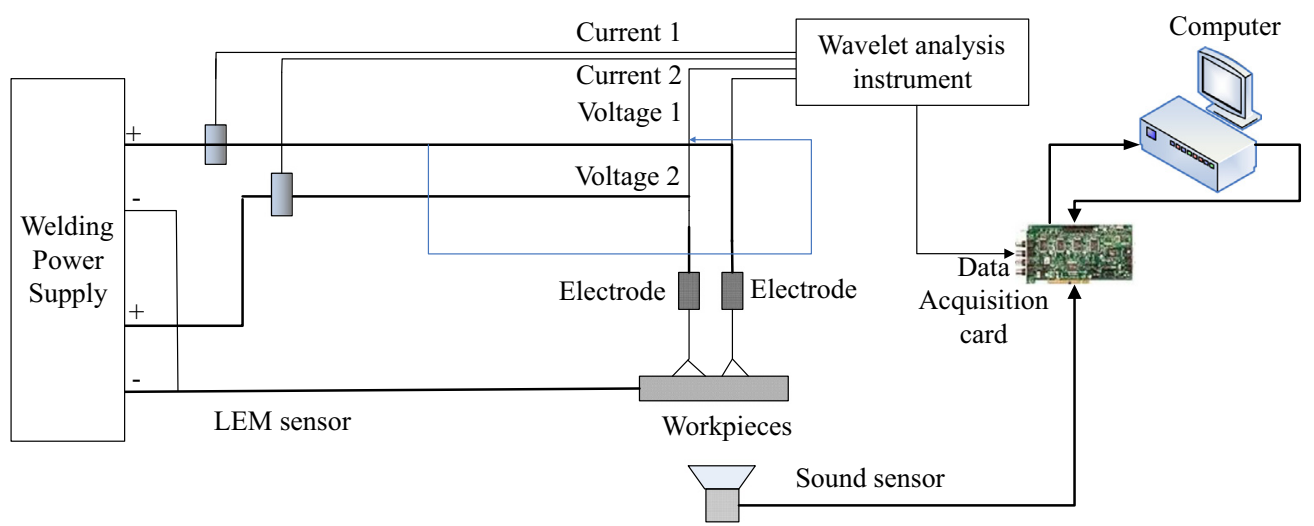

Fig. 1. Integrated experiment platform. 

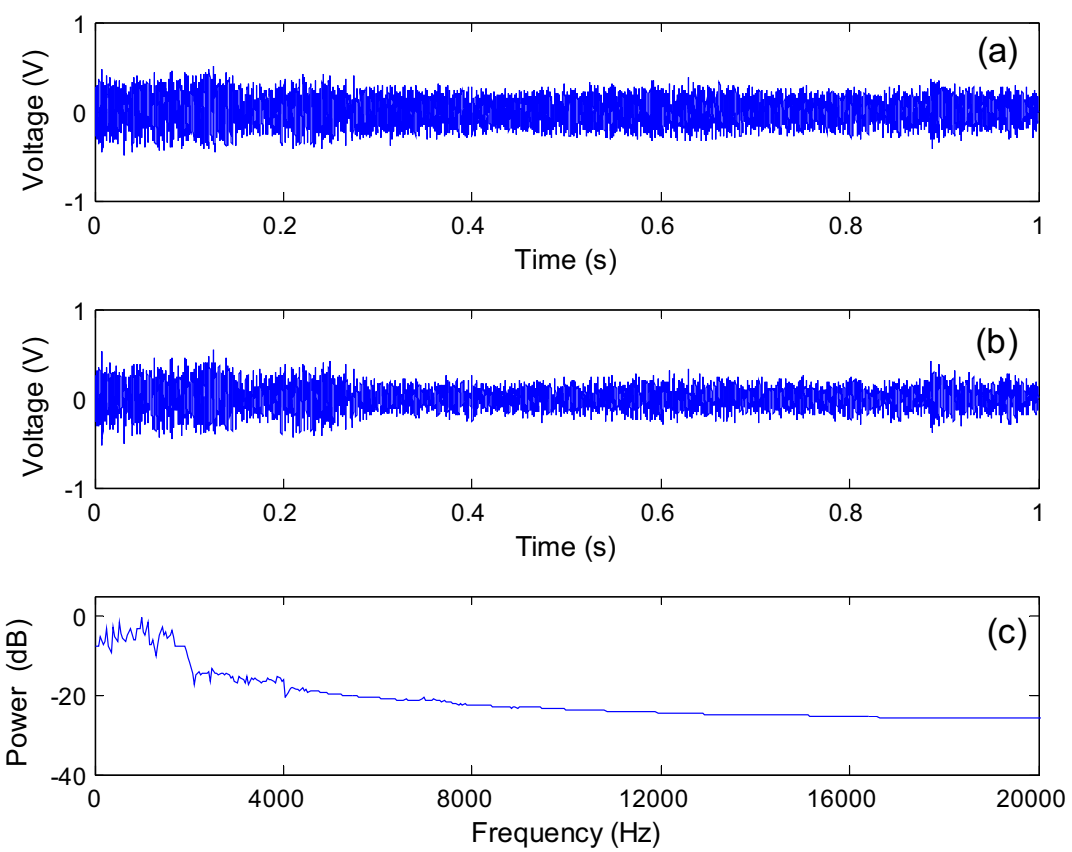

Fig. 2. Filtered signals and power spectrum analysis (a) Original arc sound signals (b) Arc sound signals filtered by wavelet packet (c) Power spectrum analysis.

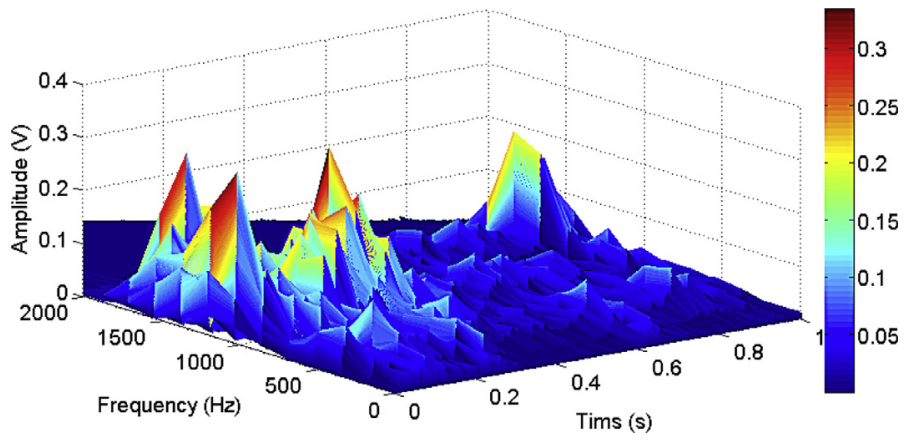

Fig. 3. Arc sound signal after STFT.

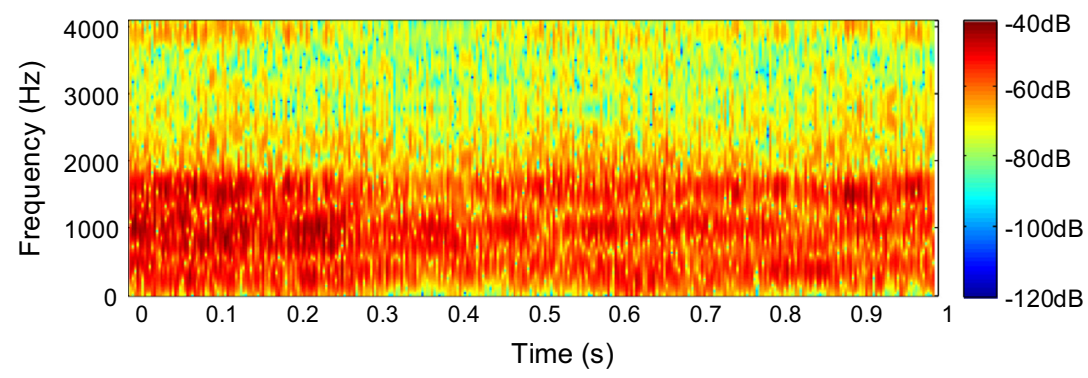

Fig. 4. Spectrogram of the arc sound signal.

consistent. Darker colors appeared at $0.9 \mathrm{~s}$ and it meant that few high PSD distributions might exist during this time. Spectrogram analysis results were consistent with the actual situations. It meant that the stability of arc sound signals could be studied by analyzing PSD variation of the spectrogram. 
When the welding process is stable, arc sound is gentle, and the corresponding arc sound waveform is regular, in the meanwhile, the max PSD has few changes and shows strong regularity on the time-frequency interface. When the welding process is unstable, the arc sound is intermittent and mixed with "poop" sounds brought by splashes and short circuits. Correspondingly, in the spectrogram, the max PSD denotes larger change appearing. More unstable the arc sound signals, more changes and irregular of max PSD can be observed.

\section{Max PSD sample entropy evaluation method}

According to the analysis of arc sound spectrogram, it could be seen that the regularity of max PSD in the time-frequency interface was directly related to the stability of arc welding process. More unstable welding process means more disorder distribution of max PSD. Entropy is a criterion which can be employed to represent the level of disorder. In this work, a new method which employs the sample entropy of arc sound max PSD distribution is proposed to evaluate the stability of arc welding process.

In this method, max PSD line in the arc sound spectrogram can be first calculated. The max PSD line, is a line which connects each max PSD point at each time interval in the time-frequency interface in the employed arc sound spectrogram. Then the corresponding coordinate and PSD value in each point in the max PSD sequence can be analyzed by sample entropy method, and the degrees of their disorders can be obtained. Finally, the arc welding process stability can be evaluated using our proposed evaluation criterion.

The detailed calculation procedures can be summarized as follows:

Step 1: Sampling all the arc sound signals during the welding process using the platform;

Step 2: Pretreating all the arc sound data, using wavelet packet filtering tool to exclude the noises;

Step 3: Plotting the spectrogram of arc sound signals, and obtaining the relation between time, frequency and PSD;

Step 4: Calculating the max PSD at each time interval by means of mathematically comparing the PSD values in the spectrogram, and then confirming the corresponding time and frequency, obtaining the max PSD line in the time-frequency interface, and then the max PSD values sequence can be obtained;

Step 5: To reduce the effect of the difference of amplitude variations on the quantitative results, average of max PSD values can be introduced, and then the max PSD values sequence in Step 4 can be calculated. The following equation can describe this procedure:

$$
P m_{a v}=\sum_{i=1}^{N} P_{\max }(i) / N,
$$

where $P_{\max }(i)$ denotes the max PSD value at each point, $N$ is the number of the sequence, $P m_{a v}$ is the average of max PSD values sequence.

Step 6: Calculating the sample entropy of max PSD [33] as follows:

A data sequence $P(i)$ based on $P m_{a v}$ is built as follows:

$$
P(i)=L_{\max }(i)^{*} P m_{a v},
$$

where $L_{\max (i)}$ represents the corresponding PSD of each point of the max PSD line, $i=1,2, \ldots N$. Then according to the method mentioned in Lake et al's work [33], the sample entropy of max PSD can be calculated as follows:

$$
P_{\text {SaEn }}=\operatorname{SampEn}(P, m, r, N) \text {, }
$$

where $P_{\text {saEn }}$ is the sample entropy of max PSD for the data sequence $P$ in Eq. (2), $m, r$ are respectively the dimension of vector and threshold for calculating the sample entropy, $\operatorname{SampEn}(P, m, r, N)$ is a proposed function by ourselves used in Matlab, the detailed mathematical description was included in our previous works [34,35].

Step 7: Calculating the standard deviation of PSD sample entropy as follows:

$$
P_{\text {std }}=\sqrt{\sum_{i=1}^{n}\left(P_{\text {SaEn }}(i)-P m_{a v}\right)^{2} /(n-1)}
$$

where $P_{\text {std }}$ denotes the standard deviation of PSD sample entropy.

Step 8: Calculating the arc sound sample entropy (ASSE) as follows:

$$
A S S E=P_{\text {SaEn }} \times P_{\text {std }} \text {. }
$$

ASSE is a proposed evaluation criterion, because $P_{\text {SaEn }}$ and $P_{\text {std }}$ have the similar variation tendency, their product can have more significant effect on evaluation.After obtaining the value of ASSE, the stabilities of different arc sound signals can be obtained by comparing their corresponding ASSEs.

\section{Experiments and analyses}

To testify the effect of evaluating the stability of arc sound signals by means of the max PSD of arc sound spectrogram, according to the operational characteristics of arc welding, four arc sound signals, which had the same operational 


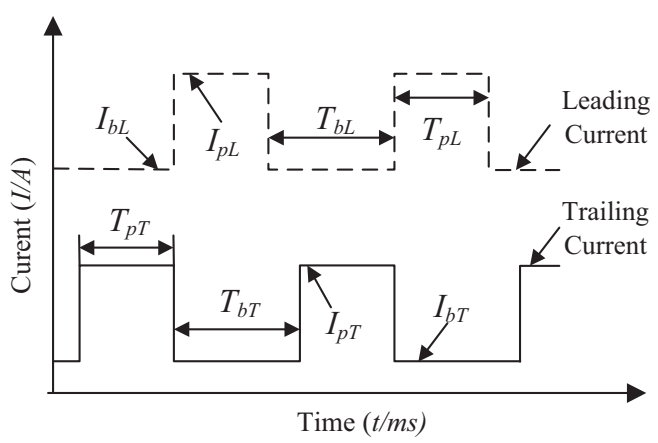

Fig. 5. Key current parameters during the experiments.

parameters but different speeds of wire feed, were chosen to do further analysis and calculation. Under this circumstance, the stabilities of the four sample signals were different. All of these four samples were collected by the platform shown in Fig. 1 . To conveniently make analysis and assure the consistency of the results, the signals in between 0 to $1 \mathrm{~s}$ after arcing were selected. Then the data would be processed by wavelet packet toolbox in Matlab 2013a.

During the experiments, there were many key current parameters to be set, such as peak current of leading wire $\left(I_{p L}\right)$, base current of leading wire $\left(I_{b L}\right)$, peak current of trailing wire $\left(I_{p T}\right)$, base current of trailing wire $\left(I_{b T}\right)$, peak time of leading wire $\left(T_{p L}\right)$, base time of leading wire $\left(T_{b L}\right)$, peak time of trailing wire $\left(T_{p T}\right)$, base time of trailing wire $\left(T_{b T}\right)$. Their detailed physical description can be shown in Fig. 5.

In addition, leading wire feed speed $\left(V_{W L}\right)$ and trailing wire feed speed $\left(V_{W T}\right)$ were also key parameters actually. Table 1 shows the corresponding values of these 10 key parameters for the four samples.

In Fig. 6 which shows the Sample 1, the amplitude of the voltage variation was very large during the process, especially at some significant stages, such as around $0.2 \mathrm{~s}, 0.4 \mathrm{~s}$ and $0.6 \mathrm{~s}$. The most significant variation appeared from $0.85 \mathrm{~s}$ to $1 \mathrm{~s}$.

The corresponding 3D figure after processed by STFT could be shown in Fig. 7. The peaks in the figure indicated sudden change of voltage of arc sound signal. The peak randomly appeared, and the voltage had large variation, which denoted that the signal was unstable with large mutation. The largest amplitude of the variation could approach $0.6 \mathrm{~V}$. The whole signal had fierce variation with many mutations which were beyond $0.2 \mathrm{~V}$. In addition, peaks appeared around $0.2 \mathrm{~s}, 0.4 \mathrm{~s}, 0.6 \mathrm{~s}$ and $0.85 \mathrm{~s}$, which corresponded to what was shown in Fig. 6.

In Fig. 8 which showed the Sample 2, the amplitude increased stage by stage, which meant that the PSD of arc sound was not uniform. The voltage of the signal increased around $0.25 \mathrm{~s}$ and $0.6 \mathrm{~s}$, whose amplitudes achieved about $0.4 \mathrm{~V}$. In summary of this figure, the voltage variation was not steady with some significant fluctuations.

Table 1

The values of parameters of the four samples used in the experiments.

\begin{tabular}{lllll}
\hline Parameter & Sample1 & Sample2 & Sample3 & Sample4 \\
\hline$I_{\mathrm{pL}}$ & 230.00 & 200.00 & 360.00 & 320.00 \\
$I_{\mathrm{bL}}$ & 130.00 & 58.00 & 91.00 & 88.00 \\
$I_{\mathrm{pT}}$ & 310.00 & 210.00 & 235.00 & 308.00 \\
$I_{\mathrm{bT}}$ & 140.00 & 115.00 & 128.00 & 75.00 \\
$T_{p L}$ & 3.70 & 3.50 & 3.70 & 3.50 \\
$T_{b L}$ & 9.00 & 8.80 & 5.10 & 3.00 \\
$T_{p T}$ & 3.70 & 3.50 & 3.70 & 13.00 \\
$T_{b T}$ & 9.00 & 8.80 & 100 & 7.81 \\
$V_{W L}$ & 6.74 & 6.29 & 1.35 & 10.03 \\
$V_{W T}$ & 8.15 & 6.02 & 10.03 & \\
\hline
\end{tabular}

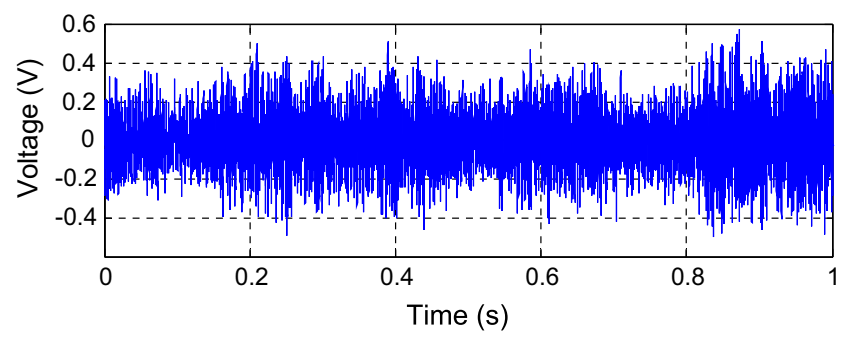

Fig. 6. Arc sound waveform figure of Sample 1 after wavelet packet filtering. 


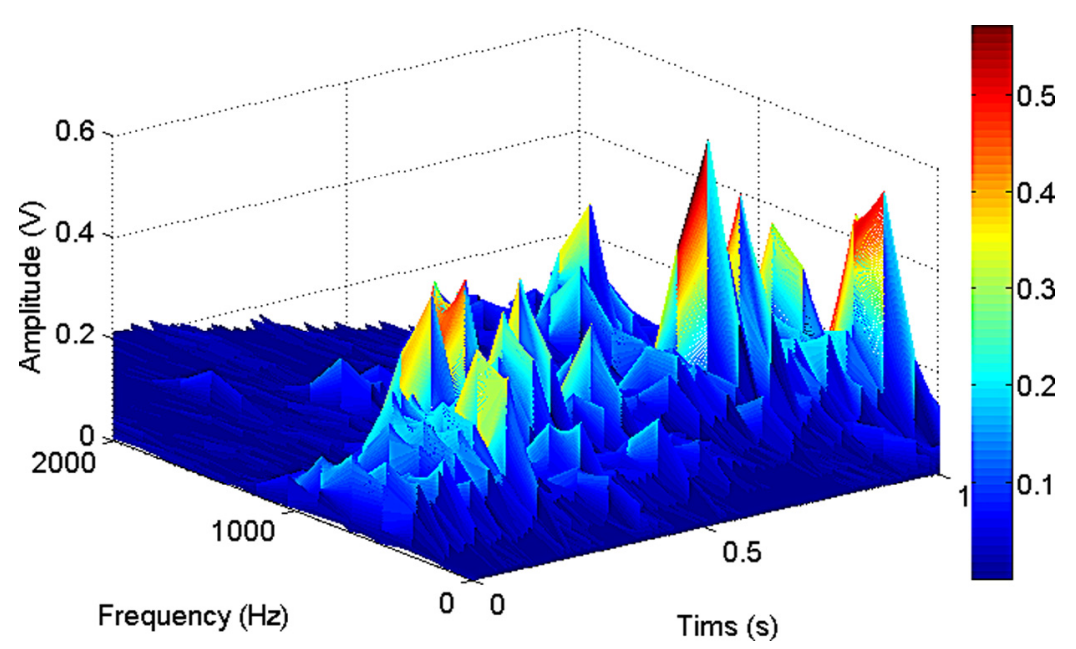

Fig. 7. 3D figure of Sample 1 after STFT processing.

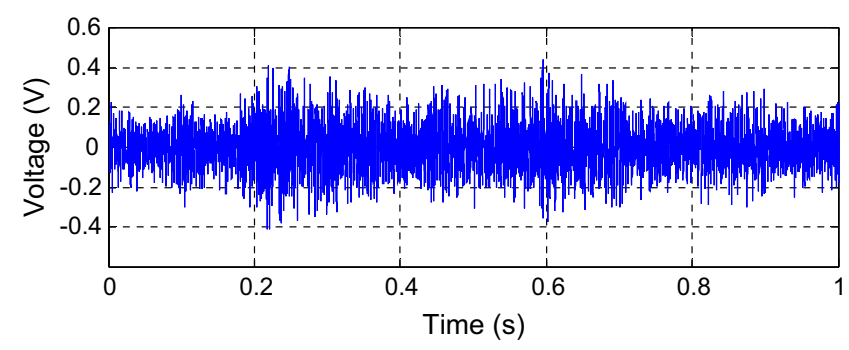

Fig. 8. Arc sound waveform figure of Sample 2 after wavelet packet filtering.

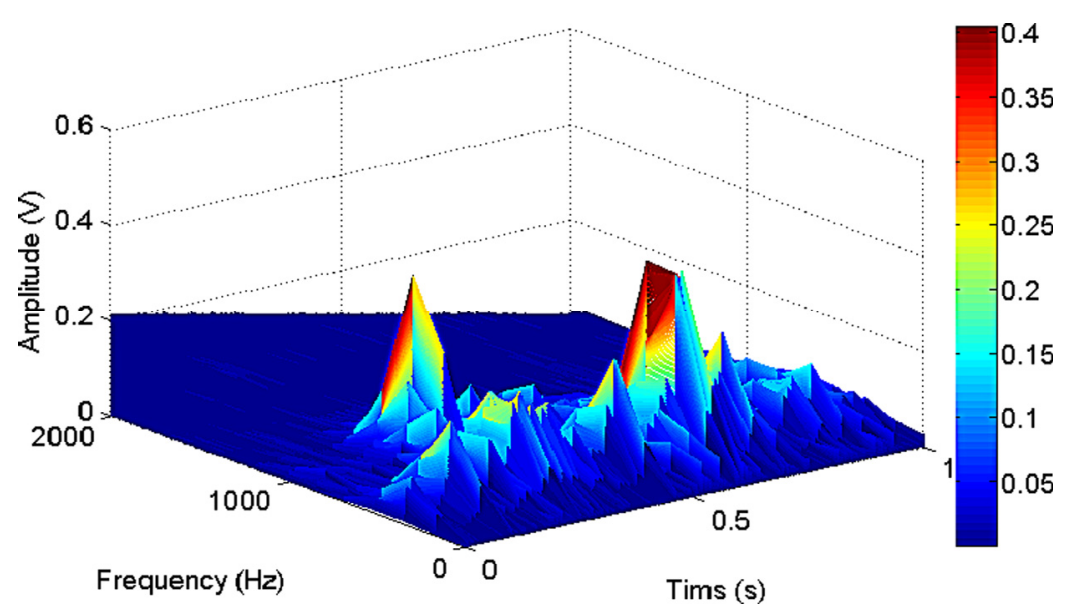

Fig. 9. 3D figure of Sample 2 after STFT processing.

Fig. 9 was the corresponding 3D figure after STFT processing. It can be found that this signal was more stable than that of Sample 1 . The large mutations only appeared around $0.25 \mathrm{~s}$ and $0.6 \mathrm{~s}$, with below $0.4 \mathrm{~V}$ of amplitude, and others were below $0.2 \mathrm{~V}$.

In Fig. 10 which showed the Sample 3, the PSD change was also gentle, only a small voltage variation appeared around $0.9 \mathrm{~s}$.

Fig. 11 was the corresponding 3D figure after STFT processing. This signal was more stable. According to the figure, majority of the voltage variations were below $0.08 \mathrm{~V}$, and no large variation appeared. Only one PSD mutation occurred around $0.9 \mathrm{~s}$, with only about $0.12 \mathrm{~V}$ of amplitude. 


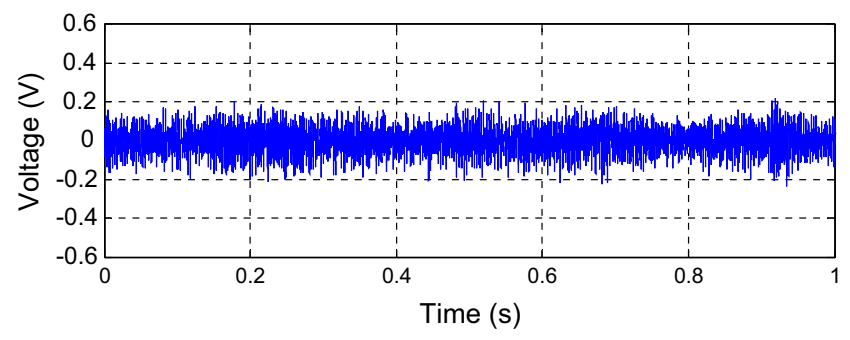

Fig. 10. Arc sound waveform figure of Sample 3 after wavelet packet filtering.

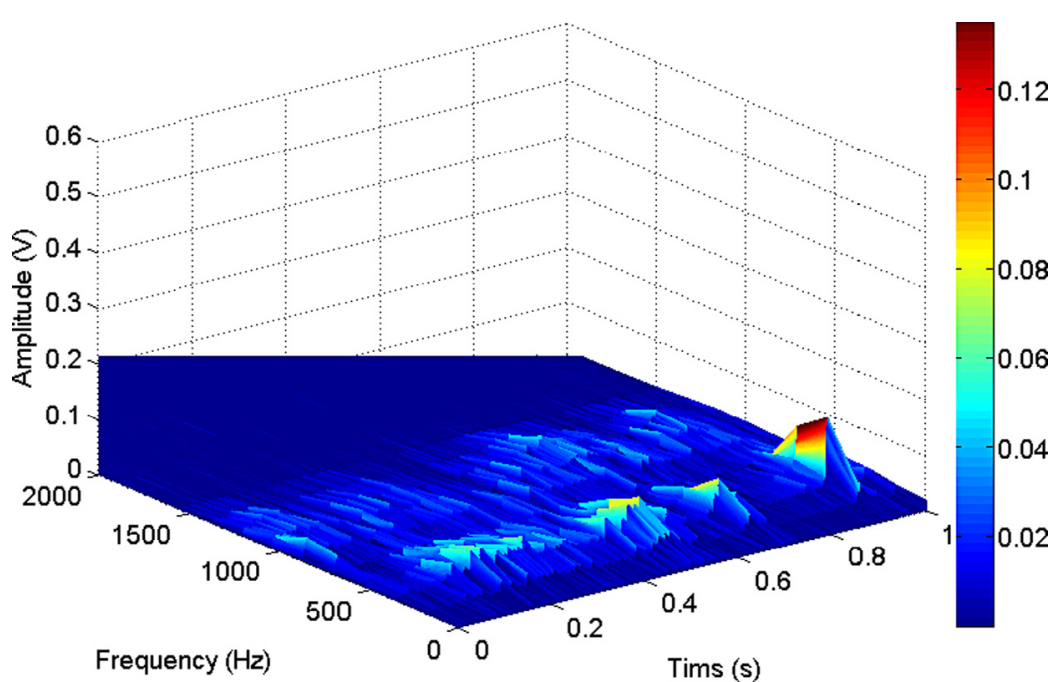

Fig. 11. 3D figure of Sample 3 after STFT processing.

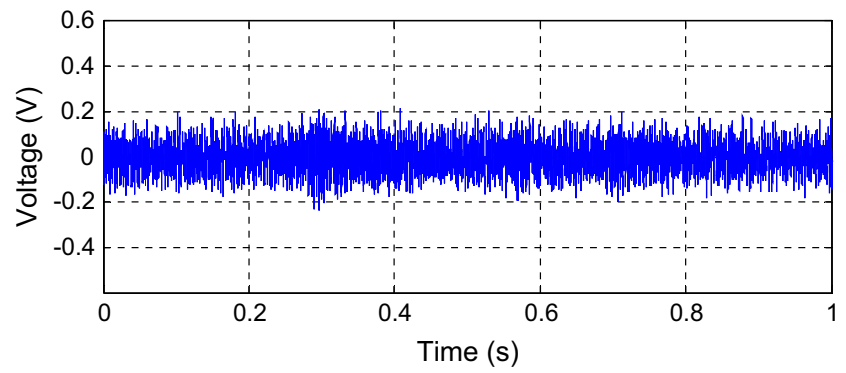

Fig. 12. Arc sound waveform figure of Sample 4 after wavelet packet filtering.

Fig. 12 showed the waveform of the Sample 4, the amplitude of arc sound was relative small, which meant that no large PSD change occurred. During the process, the sound was so gentle and no "poop" appeared. In the meanwhile, the voltage variation was gentle during all the process.

Fig. 13 was the corresponding 3D figure after STFT processing. It can be observed that this signal was the most stable one. The amplitude surface was approximately a plane. All of the amplitudes were below $0.06 \mathrm{~V}$, without significant mutation. The voltage had small variation, which denoted the signal was stable with gentle sound.

According to the above figures and corresponding analyses, it could be clearly found that the stabilities of arc sound signals were better by better from Sample1 to Sample 4.

The arc sound spectrograms of four samples were shown in Figs. 14-17. The white lines denote the max PSD lines, while blue points denote the max PSD points at the designated time and frequency.

It can be seen that the max PSD lines in Fig. 14 was much more irregular, and with large amplitude changes. While the lines in Fig. 15 was irregular, but the amplitude variation was less than that in Fig. 14; the max PSDs in Figs. 16 and 17 were more regular with large variation ranges. The variation range in Fig. 16 was a little larger than that in Fig. 17, and around 


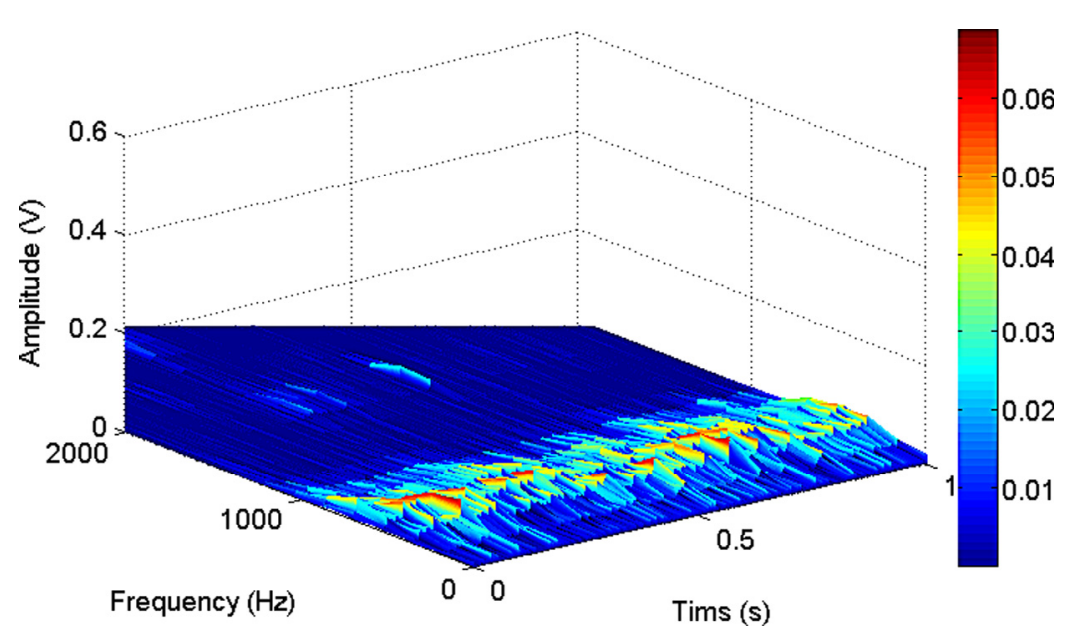

Fig. 13. 3D figure of Sample 4 after STFT processing.

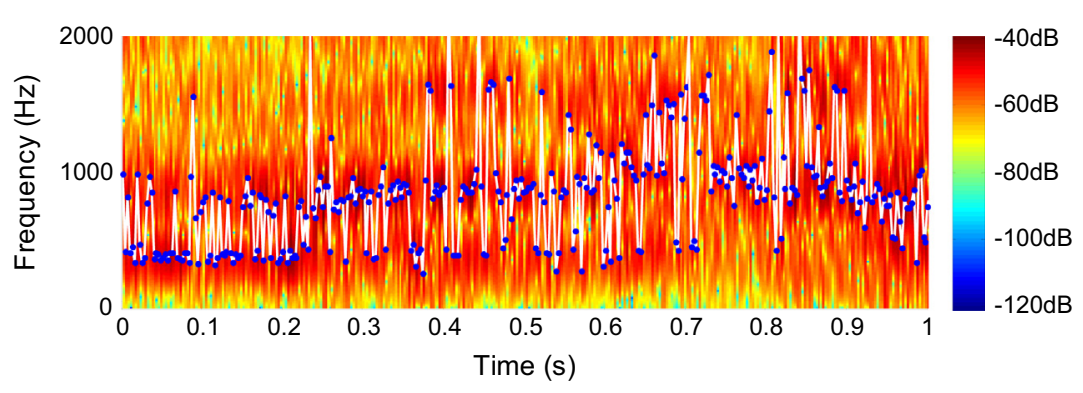

Fig. 14. Arc sound spectrograms and max PSD line of sample 1 .

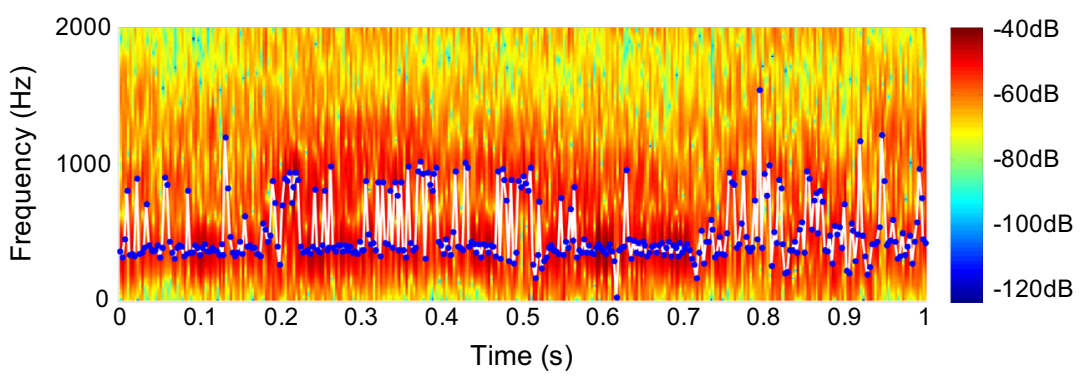

Fig. 15. Arc sound spectrograms and max PSD line of sample 2.

$0.9 \mathrm{~s}$, it was significant that the color was dark, which denoted that the PSD was very large. This phenomenon corresponded to the peak at $0.9 \mathrm{~s}$ shown in Fig. 11. Hence, according to these analyses, the stability of the welding process can be roughly deduced, however, the detailed qualitatively description cannot be obtained in this stage.

In addition, to significantly compare the four signals, four max PSD lines can be plotted together shown in Fig. 18.

According to Fig. 18, the, max PSD of Sample 1 was larger with random peaks appearing, such as around $0.1 \mathrm{~s}, 0.24 \mathrm{~s}, 0.4 \mathrm{~s}$, $0.7 \mathrm{~s}$ and $0.8 \mathrm{~s}$, and the max PSD variation was so irregular. The variation of max PSD of Sample 2 was relatively regular, however, its variation was very random with some mutations. The max PSD of Sample 3 was regular, and its variation was less than that in preceding two samples, moreover, no significant mutation appeared. The max PSD of Sample 4 was more regular, only small max PSD variation appeared around $0.34 \mathrm{~s}, 0.38 \mathrm{~s}$ and $0.56 \mathrm{~s}$. However, the variation lasted short duration and the phenomena were not clear. In summary, from this figure, it can be observed that the stability of Sample 1 was the worst, the stability of Sample 2 was only better than that of Sample 1, while the Sample 4 had the best stability. It can be concluded that the stability of different samples can be obtained according to analyze the corresponding max PSD lines. Then to obtain more reliable stability analyzing results, the method proposed in Section 3 can be employed as follows. 


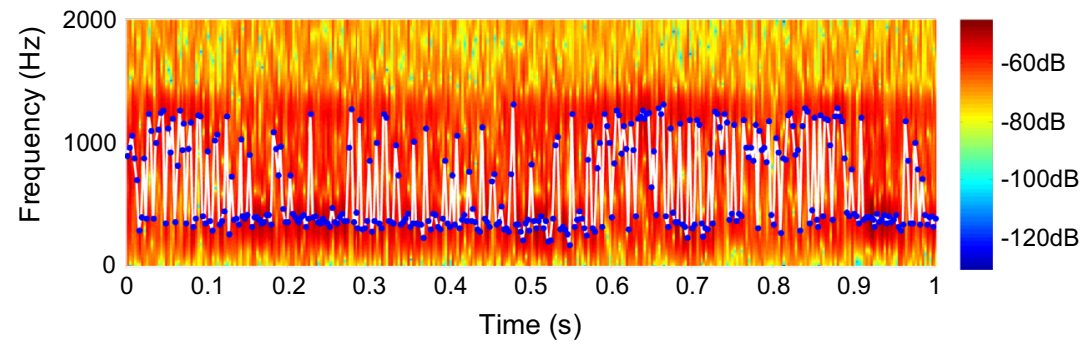

Fig. 16. Arc sound spectrograms and max PSD line of sample 3.

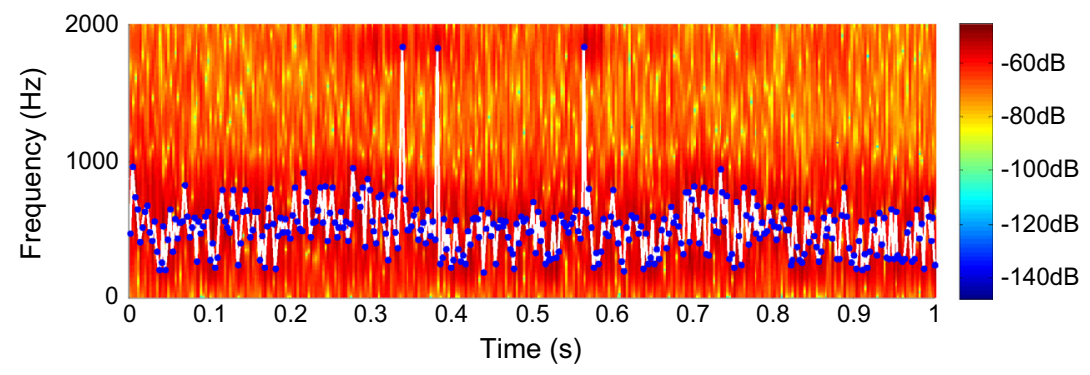

Fig. 17. Arc sound spectrograms and max PSD line of sample 4.

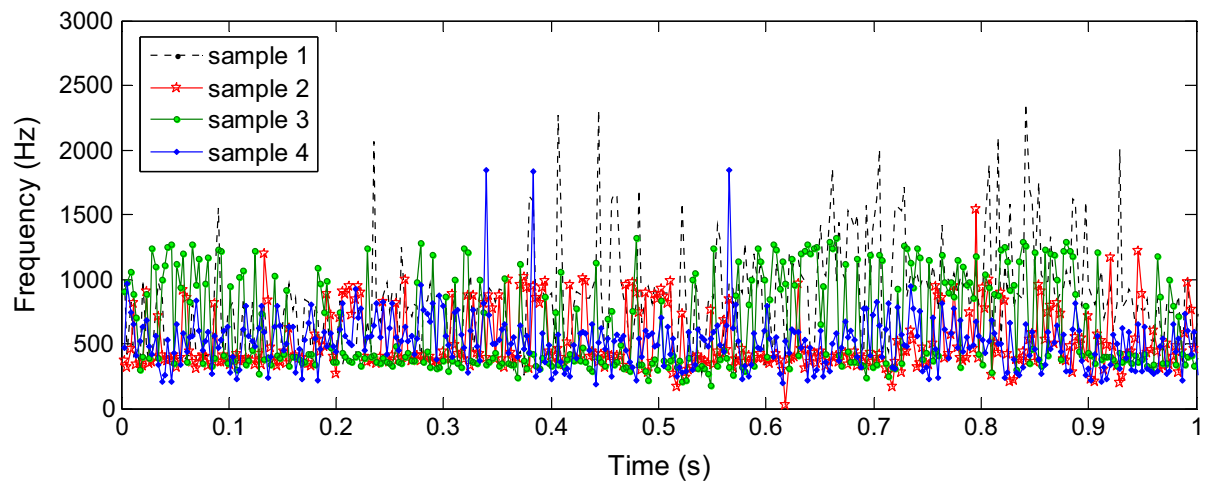

Fig. 18. Max PSD lines of four samples.

Sample entropy calculation requires determining three parameters: $m, r$ and $N$. Different sample entropies correspond to different $m$ and $r$. Lake et al. [33] thought the sample entropy distribution was normal when $m$ was smaller and $r$ was larger, and small amount of lost data points did not affect the entropy calculation. After several times' trials, we selected $m=2$, $r=0.002, N=30$ for calculation, and the results are shown in Table 2 .

According to the calculations shown in Table 2, there were little difference in the averages of max PSDs between Signal 1 and Signal 2, but $P_{\text {SaEn }}$ and $P_{\text {std }}$ of Signal 1 were about twice of those of Signal 2, which showed that there were more disorders and complexities in Signal 1 than that in Signal 2. The $P m_{a v}$ s of Sample 3 and Sample 4 had little difference, and those values were significantly lower than those of Sample 1 and Sample 2 . However, the sample entropy $\left(P_{\text {SaEn }}\right)$ of Sample 3 was significantly larger than that of Sample 4, it was because that the larger PSD variation around 0.6 s of Sample 3 made the entire

Table 2

Sample entropy evaluation results.

\begin{tabular}{|c|c|c|c|c|}
\hline Sample No & $P m_{a v}$ & $P_{\text {SaEn }}$ & $P_{\text {std }}$ & ASSE \\
\hline 1 & $2.3930 \mathrm{e}-05$ & 1.0664 & 0.0096 & 0.0102 \\
\hline 2 & $1.6762 \mathrm{e}-05$ & 0.5388 & 0.0038 & 0.0021 \\
\hline 3 & $4.8966 \mathrm{e}-06$ & 0.4977 & 0.0018 & $8.7177 e-04$ \\
\hline 4 & $5.1393 e-06$ & 0.1028 & 0.0011 & $1.0930 \mathrm{e}-04$ \\
\hline
\end{tabular}




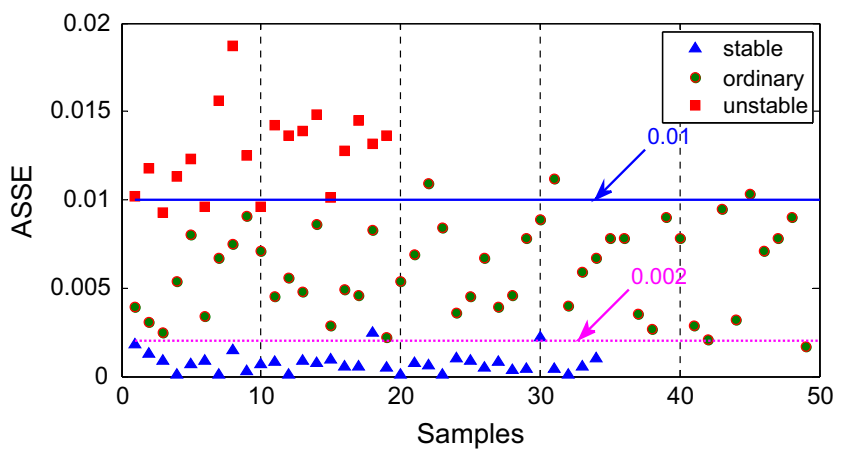

Fig. 19. Scatter of sample ASSE calculation for 100 samples.

sample entropy largely changed, and it also lead to the standard deviation of spectrogram PSD sample entropy $\left(P_{s t d}\right)$ of Sample 3 was larger than that of Sample 4. The waveform variation of Sample 4 was gentle, and the variation amplitude of max PSD was very small, moreover, the corresponding $P_{S a E n}$ and $P_{\text {std }}$ were relatively small. In addition, according to the corresponding values of ASSE, which directly showed the stability of arc sound, those values clearly showed the stability of welding process of these four samples were better by better.

According to above analyses, our proposed spectrogram PSD sample entropy method can be effectively employed to analyze the stability of arc sound signal, which can also be extensively used to analyze the stability of arc welding process. The reliable and convinced quantitative results can be obtained. To further testify the effectiveness of this method, more experiments were conducted. We collected 100 different arc sound signals, and then used the knowledge of stability of electrical signals, observation of welding process, quality of welding seams, to accomplish the evaluation of process stability. There were 19 unstable samples, 47 ordinary samples and 34 stable samples, respectively. Then the above method was employed for these samples, and each spectrogram PSD sample entropy can be correspondingly obtained.

After obtained the spectrogram PSD sample entropy of each sample, according to rule of ASSE results, three different zones were divided. When ASSEs were below 0.002, it meant that corresponding samples were stable. When ASSEs were below 0.01 and equal to or above 0.002, it meant samples were ordinary. When ASSEs were above or equal to 0.01, it meant samples were unstable. Then the scatter for 100 samples was shown in Fig. 19.

It can be seen that the distribution of ASSE followed expectance, only 10 values were not in the corresponding zones. Hence, this conclusion denoted that the accuracy rate could reach 90\%. Meanwhile, it can be found that the deviation of those 10 values were very small, which meant that the reliability of this method was very high.

\section{Conclusion and future work}

This work proposed a method of employing arc sound spectrogram to analyze the arc sound stability, and then the stability of arc welding process can be quantitatively evaluated. Corresponding experiments were conducted to verify the effectiveness of the method and algorithm. After collected the arc sound signals, the original signals were processed by STFT, and the relation between max PSD after wavelet packet filtering and stability were analyzed. It can be found that the amplitude of variation and frequency of max PSD were directly relative to the arc sound stability.

Then the max PSD value for each time interval could be calculated, and the corresponding max PSD line could be obtained. A sample entropy evaluation method for arc sound stability was designed based on the irregularity of the max PSD line. The experimental results showed that this method excluded the effect of operational parameters on arc sound, and made the difference of evaluating result for the stability of arc welding process much clearer. Satisfactory effects were obtained in many experiments. Hence, employing this method to evaluate the stability of welding process can achieve expected performance, and this work can improve the research of stability and quality evaluation during arc welding process.

In future work, we will further improve the evaluation model. In addition, more reasonable divisions of ASSE values zones for different stabilities will be conducted, so as to provide services for application of intelligent control algorithm in arc welding production.

\section{Acknowledgements}

The authors would like to thank the Foundation for Natural Science Foundation of Guangdong Province (2015A030313663), Public Welfare Research and Capacity Building Project of Guangdong Province (2015A010104010), China Postdoctoral Science Foundation (2016M602461), and Characteristic Innovation Project of Guangdong Province Ordinary University (2014KTSCX145). 


\section{References}

[1] K. Luksa, Z. Rymarski, Collection of arc welding process data, J. Achiev. Mater. Manuf. Eng. 17 (1-2) (2006) 377-380.

[2] M.J.M. Hermans, G.D. Ouden, Process behavior and stability in short circuit gas metal arc welding, Weld. J. 78 (4) (1999) 137s-141s.

[3] S. Adolfsson, A. Bahrami, G. Bolmsjö, I. Claesson, On-line quality monitoring in short-circuit gas metal arc welding, Weld. J. 78 (2) (1999) 59s-73s.

[4] A.F. Manz, Welding arc sounds, Weld. J. 60 (5) (1981) 23-27.

[5] J.F. Wang, B. Chen, H.B. Chen, S.B. Chen, Analysis of arc sound characteristics for gas tungsten argon welding, Sens. Rev. 29 (3) (2009) $240-249$.

[6] L. Liu, H. Lan, H. Zheng, X. Jian, Feature extraction and dimensionality reduction of arc sound under typical penetration status in metal inert gas welding, Chin. J. Mech. Eng. 25 (2) (2012) 293-298.

[7] M. Čudina, J. Prezelj, Evaluation of the sound signal based on the welding current in the gas-metal arc welding process, Proc. Inst. Mech. Eng. Part C J. Mech. Eng. Sci. 217 (5) (2003) 483-494.

[8] K. Pal, S. Bhattacharya, S.K. Pal, Investigation on arc sound and metal transfer modes for on-line monitoring in pulsed gas metal arc welding, J. Mater. Process. Technol. 210 (10) (2010) 1397-1410.

[9] L. Grad, J. Grum, I. Polajnar, Feasibility study of acoustic signals for on-line monitoring in short circuit gas metal arc welding, Int. J. Mach. Tools Manuf 44 (5) (2004) 555-561.

[10] D. Saini, S. Floyd, An investigation of gas metal arc welding sound signature for on-line quality control, Weld. J. 77 (4) (1998) 172s-179s.

[11] J. Dennis, H.D. Tran, H. Li, Spectrogram image feature for sound event classification in mismatched conditions, IEEE Sig. Proc. Lett. 18 (2) (2011) 130133.

[12] K. Schutte, J. Glass, Speech recognition with localized time-frequency pattern detectors, in: IEEE Workshop on Automatic Speech Recognition \& Understanding, 2007. ASRU, 2007, pp. 341-346.

[13] D.K. Mellinger, C.W. Clark, Recognizing transient low-frequency whale sounds by spectrogram correlation, J. Acoust. Soc. Am. 107 (6) (2000) 35183529.

[14] P.H. Peeling, S.J. Godsill, Generative spectrogram factorization models for polyphonic piano transcription, IEEE Trans. Audio Speech Lang. Proc. 18 (3) (2010) 519-527.

[15] O. Rioul, M. Vetterli, Wavelets and signal processing, IEEE Signal Process. Mag. 8 (1991) 14-38.

[16] C. Taishih, P. Ru, S.A. Shamma, Multiresolution spectrotemporal analysis of complex sounds, J. Acoust. Soc. Am. 118 (2) (2005) $887-906$.

[17] S.M. Pincus, I.M. Gladstone, R.A. Ehrenkranz, A regularity statistic for medical data analysis, J. Clin. Monit. 7 (4) (1991) $335-345$.

[18] S. Pincus, Approximate entropy (ApEn) as a complexity measure, Chaos 5 (1) (1995) 110-117.

[19] S.M. Pincus, Approximate entropy as a measure of system complexity, Proc. Natl. Acad. Sci. 88 (6) (1991) $2297-2301$.

[20] S. Pincus, R.E. Kalman, Irregularity, volatility, risk, and financial market time series, Proc. Natl. Acad. Sci. USA 101 (38) (2004) $13709-13714$.

[21] S.M. Pincus, A.L. Goldberger, Physiological time-series analysis: what does regularity quantify?, Am J. Physiol. Heart Circulat. Physiol. 266 (4) (1994) H1643-H1656.

[22] R.A. McKinley, L.K. McIntire, R. Schmidt, D.W. Repperger, J.A. Caldwell, Evaluation of eye metrics as a detector of fatigue, Human Fact. J. Human Fact. Ergonom. Soc. 53 (4) (2011) 403-414.

[23] C.R. Tolle, R.A. Laviolette, H.B. Smartt, K.L. Kenney, D.P. Pace, J.W. James, A.D. Watkins, T. Wood, D.L. Stoner, Is there evidence of determinism in droplet detachment within the gas metal arc welding process?, in: 6th International Conference on Trends in Welding Research, 2002 , pp $380-385$.

[24] B. Cao, Y.-P. Xiang, X.-Q. Lv, M. Zeng, S.-S. Huang, Approximate entropy-a new statistic to quantify arc and welding process stability in short-circuiting gas metal arc welding, Chin. Phys. B 17 (3) (2008) 865-877.

[25] B. Cao, X.-Q. Lv, M. Zeng, Z.-M. Wang, S.-S. Huang, Approximate entropy analysis of current in short-circuiting arc welding, Acta Phys. Sin. 55 (4) (2006) 1696-1705 (in Chinese).

[26] J. Nie, Y. Shi, J. Huang, D. Fan, Approximate entropy GRNN forecast for aluminum alloy pulsed MIG welding stability, Trans. China Weld. Inst. 31 (8) (2010) 77-80 (in Chinese).

[27] W.-C. Zhang, L.-J. Yang, X.-Q. Lv, Approximate entropy based meso-spray transfer analysis of Al-alloy pulsed metal inert-gas welding under selfadapting control, Acta Phys. Sin. 60 (2) (2011) 020601.

[28] J.S. Richman, J.R. Moorman, Physiological time-series analysis using approximate entropy and sample entropy, Am. J. Physiol. Heart Circul. Physiol. 278 (6) (2000) h2039-h2049.

[29] P. Yao, Intelligent Control Strategies and Performance Evaluation of Integrated Double Wire Arc Welding Power Source PhD, South China University of Technology, Guangzhou, 2012 (in Chinese).

[30] P. Duhamel, M. Vetterli, Fast Fourier transforms: a tutorial review and a state of the art, Sig. Proc. 19 (4) (1990) $259-299$.

[31] M. Frigo, S.G. Johnson, FFTW: an adaptive software architecture for the FFT, in: Proceedings of the 1998 IEEE International Conference on Acoustics, Speech and Signal Processing, 1998, pp. 1381-1384.

[32] A. Oppenheim, R. Schafer, J. Buck, Discrete time Signal Processing, vol. 5, Prentice Hall, Upper Saddle River, 1999.

[33] D.E. Lake, J.S. Richman, M.P. Griffin, J.R. Moorman, Sample entropy analysis of neonatal heart rate variability, Am. J. Physiol. Regul. Integr. Compar. Physiol. 283 (3) (2002) R789-R797.

[34] P. Yao, J. Xue, K. Zhou, XiaoJun Wang, Sample entropy-based approach to evaluate the stability of double-wire pulsed MIG welding, Math. Prob. Eng. 2014 (2014), pp. 869631.1-8.

[35] P. Yao, Intelligent Control Strategies and Performance Evaluation of Integrated Double Wire arc Welding Power Source Doctor of Engineering, School of Mechanical and Automotive Engineering, South China University of Technology, Guangzhou, China, 2012. 